\title{
Target Localization with a Single Antenna via Directional Multipath Exploitation
}

\author{
Ali H. Muqaibel, ${ }^{1}$ Moeness G. Amin, ${ }^{2}$ and Fauzia Ahmad ${ }^{2}$ \\ ${ }^{1}$ Electrical Engineering Department, King Fahd University of Petroleum \& Minerals (KFUPM), P.O. Box 1734, \\ Dhahran 31261, Saudi Arabia \\ ${ }^{2}$ Center for Advanced Communications, College of Engineering, Villanova University, 800 E. Lancaster Avenue, \\ Villanova, PA 19085, USA
}

Correspondence should be addressed to Ali H. Muqaibel; muqaibel@kfupm.edu.sa

Received 29 September 2014; Accepted 16 December 2014

Academic Editor: Ahmad Safaai-Jazi

Copyright (C) 2015 Ali H. Muqaibel et al. This is an open access article distributed under the Creative Commons Attribution License, which permits unrestricted use, distribution, and reproduction in any medium, provided the original work is properly cited.

Target localization in urban sensing can benefit from angle dependency of the pulse shape at a radar receiver antenna. We propose a localization approach that utilizes the embedded directivity in ultra-wideband (UWB) antennas to estimate target positions. A single radar unit sensing operation of indoor targets surrounded by interior walls is considered, where interior wall multipaths are exploited to provide target cross-range. This exploitation assumes resolvability of the multipath components, which is made possible by the virtue of using UWB radar signals. The proposed approach is most attractive when only few multipaths are detectable due to propagation obstructions or owing to low signal-to-noise ratios. Both simulated and experimental data are used to demonstrate the effectiveness of the proposed approach.

\section{Introduction}

Multipath propagation arises in urban and through-the-wall radar sensing due to electromagnetic (EM) interactions of targets of interest with surrounding objects and surfaces. This leads to highly cluttered images of many ghost (false) targets, thereby compromising image quality and interpretation.

Two approaches have been proposed in the literature for dealing with multipath, namely, multipath mitigation [1] and exploitation [2]. Both approaches aim at producing a ghost-free image. Unlike the mitigation techniques, the goal of multipath exploitation methods is not to attenuate or suppress multipath returns but rather use their energy to aid in the detection, localization, and classification of the corresponding target. Multipath exploitation, however, usually requires prior knowledge of the location of major scatterers in the radar field of view. To this end, access to city maps may suffice for outdoor target detection and tracking in urban canyons. For targets inside enclosed structures, this situation requires information on interior building layouts, which, if not available in the form of blueprints, can be determined from prior surveillance operations. It was shown in [3] that it is possible to exploit multipath propagation for target localization without prior knowledge of the location of the scatterers.

Multipath exploitation has been employed in urban environments. Specular multipath returns and their Doppler spread have been utilized for enhanced moving target detection and tracking in urban canyons, especially in the absence of direct line-of-sight (LOS) paths [2, 4-7]. Multipath has also been used for image enhancement of stationary indoor scenes $[8,9]$. By properly modeling the indirect propagation paths, the energy in the multipath returns can be attributed to their respective targets, allowing a ghost-free image and an increased target-to-clutter-and-noise ratio. Further, shadowed regions of spatially extended targets were revealed via their multipath returns in $[10,11]$, whereas an inverse scattering based multipath exploitation approach was presented in [12] for imaging stationary targets with enhanced cross-range resolution. Offerings of multipath exploitation for target classification in through-the-wall radar imaging applications were examined in [13]. It is noted that the aforementioned approaches dealing with stationary targets require a radar system comprising a real or synthesized array aperture. 
Multipath exploitation for sensing indoor stationary scenes can be performed using a single-antenna ultrawideband (UWB) radar system. Deploying a physical or synthesized array aperture could be costly and logistically difficult compared to a less expensive, more flexible, and mobile single-antenna radar system based operation. In this case, relying on only target radar returns does not enable coarse or accurate cross-range estimations. However, EM interactions between targets and surrounding walls generate relatively strong multipath returns that are detectable at the receiver. The concept of exploitation is embedded in using the resolvable multipath to create virtual radar units at different locations dictated by the positions of both targets and walls. Incoherent localization can then proceed using constantrange contours corresponding to monostatic and bistatic twoway propagation between the actual radar unit, the targets, and the virtual radar units.

While the above single-antenna radar system based multipath exploitation concept was already discussed in our earlier work [14], this paper offers a new and alternative approach for target localization. The proposed approach uses a fundamental property of UWB antennas, which has been thus far overlooked in the underlying application. In essence, the transmitted and/or received signal waveforms at different angles assume different shapes [15-17]. Since UWB propagation is antenna-dependent, it contributes to target localization in multipath by (a) tagging multipath by their angles of arrivals which can be achieved by matched filters of the different pulse shapes of radar returns and (b) lowering the required number of multipath returns for target localization by discarding false solutions whose corresponding pulse shapes deviate from those associated with the respective directions. In this paper, we focus on the latter property (b) and show that the availability of one single-bounce multipath component, in addition to the direct return, is the only condition for localization. This relaxes the requirements of previously published wall association algorithm in [14], which assume the resolvability of all singleand double-bounce components.

We formulate the multipath propagation of a target positioned within a nominal rectangular room. The merits of using the proposed approach are demonstrated by showing a reduction in localization error when the directivity property is applied. Both simulations and experiments are used to evaluate the performance of the proposed approach.

The remainder of this paper is organized as follows. Section 2 presents the multipath propagation model and scene. In Section 3, the concept of directivity is introduced and verified with radar reflection measurements. The procedure for localization is introduced next where we discuss the number of possible target locations and use directivity to select among the candidates. Error analysis and discussion of results are presented in Sections 5 and 6, and conclusions are presented in Section 7.

\section{Multipath Propagation Model}

The multipath model and the geometry of the problem are depicted in Figure 1. The target is located at position $\left(-x_{t}, y_{t}\right)$

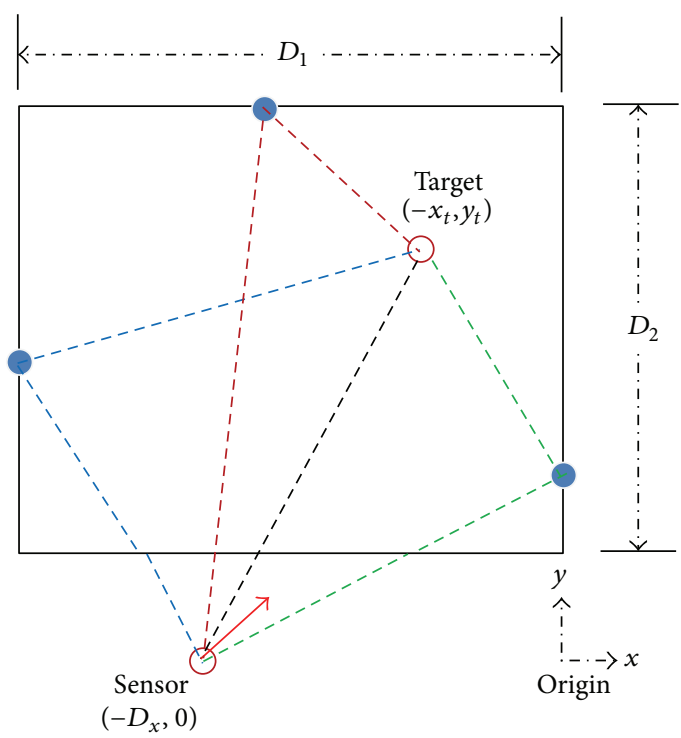

FIgURE 1: Multipath model and scene.

within a rectangular room. The right side wall is located at $x=$ 0 . The length of the side walls is $D_{2}$ meters, whereas the front and back walls have an extent of $D_{1}$ meters. The thickness and the electrical properties of the front wall are assumed to be known and their effect on signal propagation can be accounted for. The sensor is located at $\left(-D_{x}, 0\right) \mathrm{m}$. The arrow at the sensor depicts the antenna direction. The azimuth angle is measured from the $x$-axis. To keep the problem in two dimensions, the elevation angle is fixed at 0 degrees measured from the $x$ - $y$ plane.

We consider a total of seven paths by which the signal can travel from the sensor to the target and back. The first is the direct path, which does not involve any secondary reflection at an interior wall. Three single-bounce multipath components involving a reflection at an interior wall either on transit to or back from the target are assumed. We also consider three double-bounce multipath arrivals resulting from transmission and reception along the same path containing a secondary wall reflection. Additional doublebounce and higher order multipath components can also be considered and incorporated in the model. However, realdata experiments have shown that such reflections exhibit severe attenuations making them difficult to detect, and, as such, they are ignored in this paper [14].

The side walls are assumed to be perfect reflectors. When the radar transmits a pulsed signal, $s(t)$, the received signal, $r(t)$, is a superposition of the direct path and the multipath returns and can be expressed as

$$
r(t)=\sum_{i=1}^{N} A_{i} s_{i}\left(t-\tau_{i}\right)+n(t),
$$

where $N$ is the total number of signal components and $A_{i}$ and $\tau_{i}$, respectively, represent the amplitude and delay of the $i$ th component. The $i$ th signal component is denoted by $s_{i}(t)$, suggesting that the returns are not just delayed and scaled version of the transmit pulse but can also assume different 


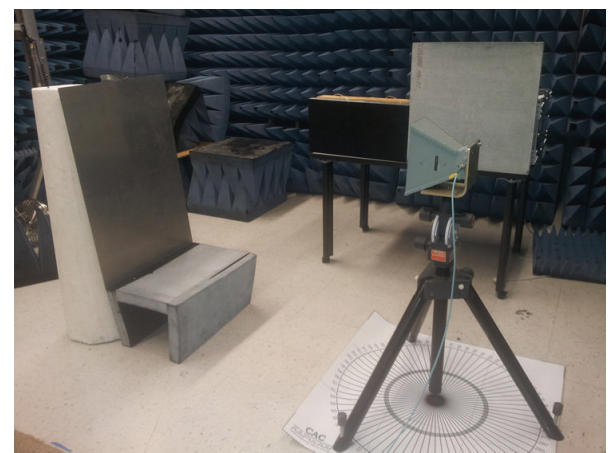

FIGURE 2: Experimental setup for the evaluation of directivity of single antenna in radar applications.

shapes. The shape-angle dependency of multipath returns is discussed in Section 3. We assume that the components of the radar return in (1) are resolvable. More specifically, the time-of-arrival (TOA) estimates for the first two arrivals, with the first one corresponding to the direct path, are readily available.

\section{Angle-Pulse Shape Dependency}

The frequency response of a single antenna varies across the antenna field of view. This phenomenon is validated through both simulation and experimentation.

For simulation, a simulator, originally developed with two antennas for communications applications [15], is redesigned to accept the wall configuration, antenna position, and polarization as simulation parameters. It is further modified to allow for oblique incidence at the receiver based on the derivation in [16]. This simulator, with the same antenna parameters as in [15], is used to generate the synthesized data.

For experimentation, a single-antenna sensor was also implemented in the Radar Imaging Lab, Villanova University, using a vector network analyzer (VNA) connected to a ridged horn antenna mounted on a tripod. The VNA operated in a stepped frequency mode with 1601 frequency points covering the 1 to $8 \mathrm{GHz}$ frequency range. The antenna was rotated to different angles from 0 degrees to 180 degrees, with 90 degrees representing normal incidence in steps of 15 degrees. A flat metal plate was used as the target. The setup is shown in Figure 2. A controlling computer recorded the amplitude and phase of the signal received by the VNA at each frequency step and each antenna angle. The acquired data was conditioned and processed to retrieve the real channel impulse response for each antenna angle. The received signal was then obtained by convolving the channel impulse response with a Gaussian input signal with sigma = $0.1 \mathrm{~ns}$. The experiment was conducted for both the horizontal and vertical polarization.

After eliminating the ringing effect from the antenna by time gating, the received signals corresponding to six different angles are depicted in Figure 3 for the case of horizontal polarization. We observe that not only there are changes in amplitudes, but also both the TOA and the shape

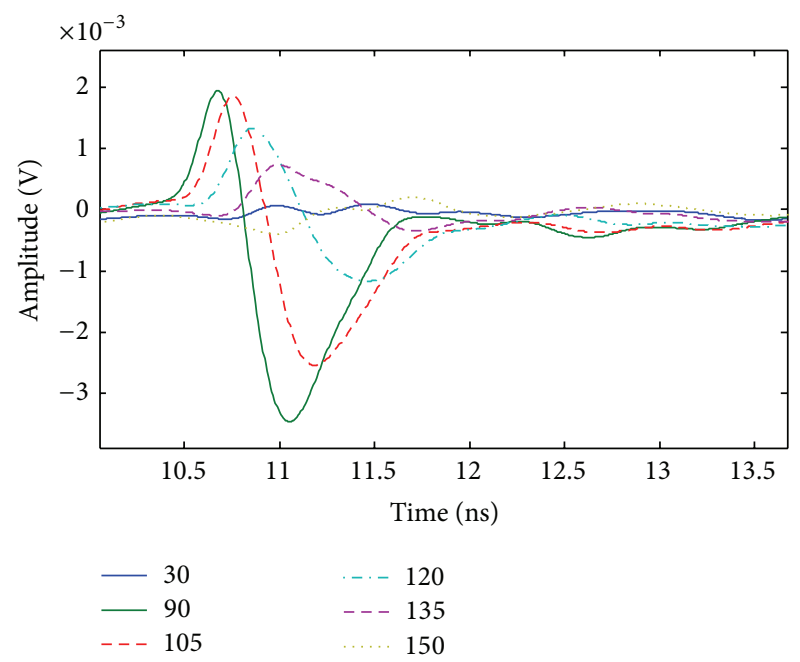

FIGURE 3: Variation of the received signal with the antenna angle for horizontal polarization.

of the signals vary with angle. The received signal for the 90degree angle is a Gaussian doublet. Comparing the returns for 30 and 150 degrees, we observe a clear difference in polarity. For the case of vertical polarization (not shown) similar variations are observed. However, one cannot discern between clockwise and counterclockwise angles relative to the 90-degree case. Consequently, the received signal at 75 degrees appears identical to the signal received at 105 degrees.

We assume accurate TOA estimates and focus on exploitation of directional variation to provide coarse angleof-arrival estimation, as delineated below. We maintain that the embedded directivity in the received signal can be utilized to improve TOA estimation by applying angle-dependent matched filters. This is, however, beyond the scope of this paper.

\section{Localization Procedure}

In this section, we incorporate pulse-directivity dependence in the localization procedure. The TOAs of all radar returns are ordered in ascending order and represented in vector form as $\tau=\left[\tau_{1}, \tau_{2}, \ldots, \tau_{7}\right]^{T}$. It is clear that the first arrival must represent the direct path, whereas the second arrival must correspond to a single-bounce multipath component. Based on the first arrival of a monostatic configuration, the location of the target is confined to an arc represented by the circle centered at the radar position and having a radius of $\tau_{1} / 2 c$, where $c$ is the wave propagation speed in free space (see Figure 4). The position of the target must satisfy the circle equation given by

$$
\left(x+D_{x}\right)^{2}+y^{2}-\frac{\tau_{1}^{2} c^{2}}{4}=0,
$$

where $-D_{1}<x<0$ and $0<y<D_{2}$.

The second TOA corresponds to a bistatic configuration involving the true and virtual sensors. Due to the multipath and wall geometry, three virtual sensors are created; two 


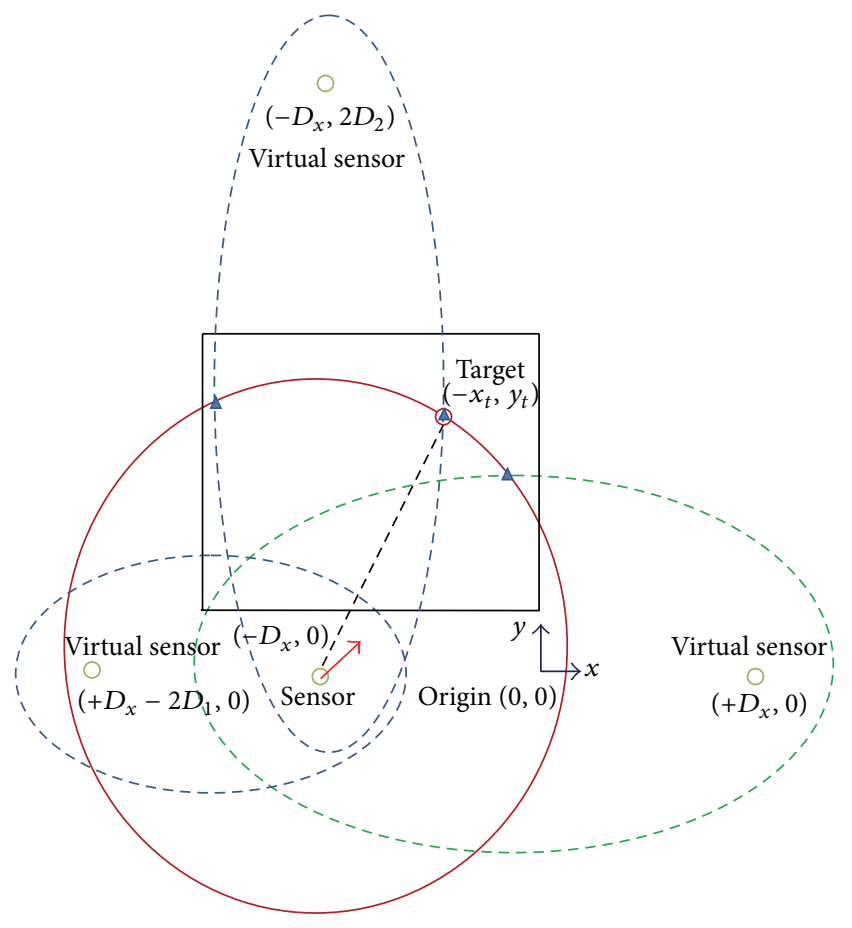

Figure 4: Candidate target locations.

sensors are due to the multipaths from the side walls, and they are located at $\left(+D_{x}, 0\right)$ and $\left(+D_{x}-2 D_{1}, 0\right)$, whereas the third virtual sensor is due to the back wall, and it is located at $\left(-D_{x}, 2 D_{2}\right)$. The target location in this case is confined to an ellipse with foci at the true and virtual sensor locations. The equations for the three possible ellipses are given by

$$
\begin{gathered}
\frac{4 x^{2}}{\tau_{2}^{2} c^{2}}+\frac{4 y^{2}}{\tau_{2}^{2} c^{2}-4 D_{x}^{2}}-1=0, \\
\frac{4\left(x+D_{x}\right)^{2}}{\tau_{2}^{2} c^{2}-4 D_{2}^{2}}+\frac{4\left(y-D_{2}\right)^{2}}{\tau_{2}^{2} c^{2}}-1=0, \\
\frac{4\left(x+D_{1}\right)^{2}}{\tau_{2}^{2} c^{2}}+\frac{4 y^{2}}{\tau_{2}^{2} c^{2}-4\left(D_{1}-D_{x}\right)^{2}}-1=0 .
\end{gathered}
$$

In general, a circle can intersect with an ellipse at 4 possible points. However, due to the underlying nature of the problem, an ellipse corresponding to the side wall can only intersect with the circle corresponding to the direct path at one valid location, while that of the back wall can result in two possible solutions. This reduces the maximum number of possible solutions to four, when considering pairs of arrivals one at time, including the direct path. More specifically, when the second arrival is due to a reflection from the right side wall, by solving (2) with (3) and dropping the unrealistic solutions, we obtain

$$
x=\frac{\left(\tau_{2}^{2} c^{2}\right) / 4}{D_{x}}\left(\frac{\tau_{1}}{\tau_{2}}-1\right) .
$$

Similarly, by solving (2) and (5), we find the solution corresponding to the involvement of left wall:

$$
x=\frac{-D_{1}^{2}+D_{x} D_{1}+\left(c^{2} \tau_{2}^{2} / 4\right)-\left(\tau_{1} \tau_{2} c^{2} / 4\right)}{D_{1}-D_{x}} .
$$

In both cases, the associated $y$ coordinate is found by substitution of the $x$-values in (2). For the back wall case, we solve (2) with (4) and obtain

$$
\begin{gathered}
y=\frac{\left(D_{2}^{2}-\left(c^{2} \tau_{2}^{2} / 4\right)+\left(\tau_{1} \tau_{2} c^{2} / 4\right)\right)}{D_{2}}, \\
x= \pm \sqrt{\frac{\tau_{1}^{2} c^{2}}{4}-y^{2}-D_{x} .}
\end{gathered}
$$

Note that $x$ can have two values.

In Figure 4, the sensor positions are indicated by small circles, while the above possible solutions are represented by small triangles. This example has only three possible solutions as the TOAs, $\tau_{1}$ and $\tau_{2}$, cannot correspond to a reflection from the left side wall. To maintain a candidate target location, the major and the semimajor axes of the corresponding ellipses must assume positive coordinate values. It is noted that in the very special case where the target exists colinearly between the real and virtual sensor corresponding to the back wall the equation of the ellipse reduces to that of a line, leading to a single solution. The lower limit on $\tau_{2}$ for a solution to exist due to multipath from the right, back, and left walls, respectively, can be expressed as $2 D_{x} / c, 2 D_{2} / c$, and $2\left(D_{1}-D_{x}\right) / c$.

We hypothesize that the second arrival belongs to a multipath from each of the three surrounding walls (side and back walls) and find the point(s) of intersection, or target location, between the corresponding ellipse and the direct path circle. In this case, the number of valid solutions is dependent on the radar location. Figure 5 illustrates the number of possible solutions within the scene for different antenna positions with $D_{1}=15 \mathrm{~m}$ and $D_{2}=20 \mathrm{~m}$. Figure 5(a) corresponds to the radar located at $(-12,0) \mathrm{m}$, whereas the radar position is $(-10,0) \mathrm{m}$ for Figure $5(\mathrm{~b})$. The results have been generated by an exhaustive search with steps of $0.5 \mathrm{~m}$. In Figure 5(a), there is a region with a single candidate solution for target location. On the other hand, no such region is present in Figure 5(b). This is because the antenna in this case is centered between the side walls, that is, $D_{x}=D_{1} / 2$, which introduces symmetry. As such, there are always multiple possible solutions.

First, we use the time of the first two arrivals to determine the candidate solutions. To explain the procedure, consider the example where the target is located at $(-6.5,11.5) \mathrm{m}$ and the radar is located at $(-12,0) \mathrm{m}$. The direct reflection arrives at $\tau_{1}=84.98 \mathrm{~ns}$ and the TOA of the second arrival corresponding to a single-bounce multipath component is 106.83 ns. Figure 5(a) suggests that there are four possible target location solutions for this scenario. The arrow at the sensor depicts the antenna direction. Figure 6 illustrates the four candidate target positions. The candidate solution marked with 3 is the true target position. 


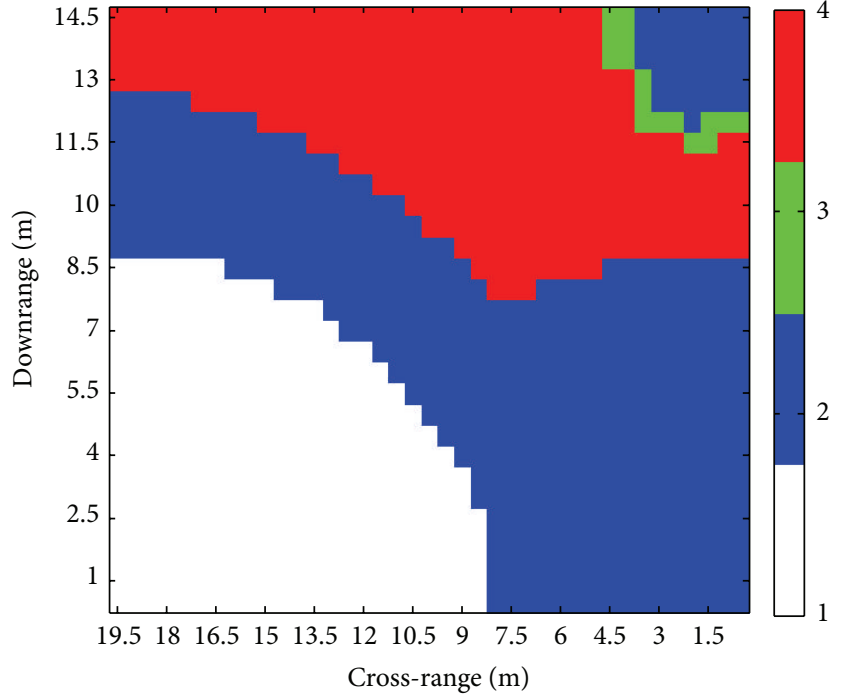

(a)

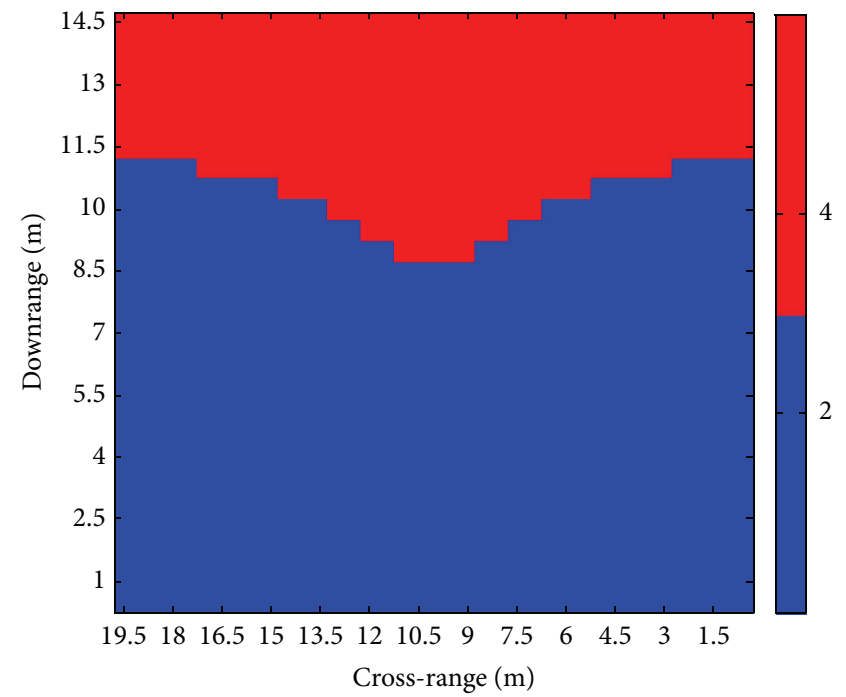

(b)

Figure 5: Number of possible solutions based on first and second time of arrivals ((a) radar $(-12,0) \mathrm{m},(\mathrm{b})$ radar $(-10,0) \mathrm{m})$.

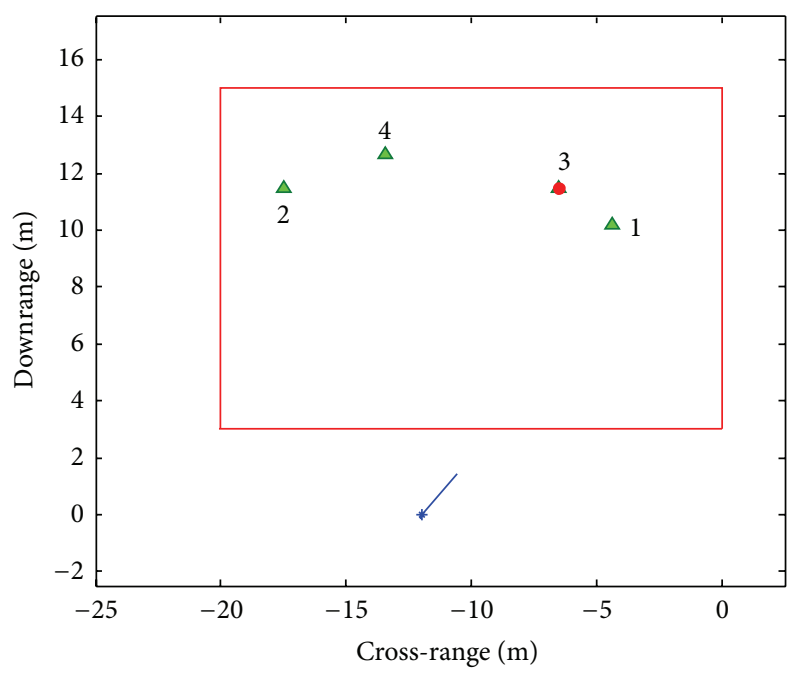

FIgURE 6: Possible candidates and considered geometry with target at $(-6.5,11.5)$.

Next, we use the directivity inherent in the UWB antenna to select the most probable target location amongst the candidate solutions. The multipath scenario corresponding to each candidate solution is reproduced. If knowledge of antenna characteristics is assumed when reproducing the candidate profiles, we refer to the procedure as directional estimation. The nondirectional estimation is then based on the assumption that all multipath components have the same pulse shape as the one received at the boresight direction. Figure 7 shows the actual radar return and the simulated directional profiles for the candidate solutions 1 through 4 of Figure 6 . The correlation between each directional synthesized return and the actual return is performed. For the considered scenario with $\mathrm{SNR}=10 \mathrm{~dB}$ and in the presence of +50 ps time jitter in first arrival and -50 ps in the second arrival the normalized correlation values are $-0.15,0.018$, 0.704 , and 0.024 for the four candidates. The highest value identifies the most probable target position.

The highest correlation results in the third candidate solution to be selected as the estimated target location. It is worth noting that the simulated returns corresponding to candidate solutions 2 and 4 have arrivals that occur before $\tau_{2}$. If we are able to further strengthen our assumption that no multipath components are missed, then these two solutions can be dropped even before the correlation step.

Although we employed a specific horn antenna for this example, in many cases only coarse angle estimation is required to choose among the possible solutions and the same procedure can be extended to other antennas.

\section{Sensitivity to Timing Errors}

In addition to the geometry of the room and the angle between the antenna and the target, the estimated position depends on the time of arrival of the direct and the multipath components, $\tau_{1}$ and $\tau_{2}$. In this section, we quantify the impact of the error in estimating the time of arrivals, $\Delta \tau_{1}$ and $\Delta \tau_{2}$, on the final estimated position $(x+\Delta x, y+\Delta y)$ and how that affects the angle required for directional estimation. The amount of error in the position estimation is evaluated as

$$
\begin{aligned}
& \Delta x=\frac{\partial x}{\partial \tau_{1}} \Delta \tau_{1}+\frac{\partial x}{\partial \tau_{2}} \Delta \tau_{2} \\
& \Delta y=\frac{\partial y}{\partial \tau_{1}} \Delta \tau_{1}+\frac{\partial y}{\partial \tau_{2}} \Delta \tau_{2}
\end{aligned}
$$

The derivations of the partial derivatives for the three cases are provided in Table 1. The error on the direction of arrival can be calculated as the angle change due to the radar 

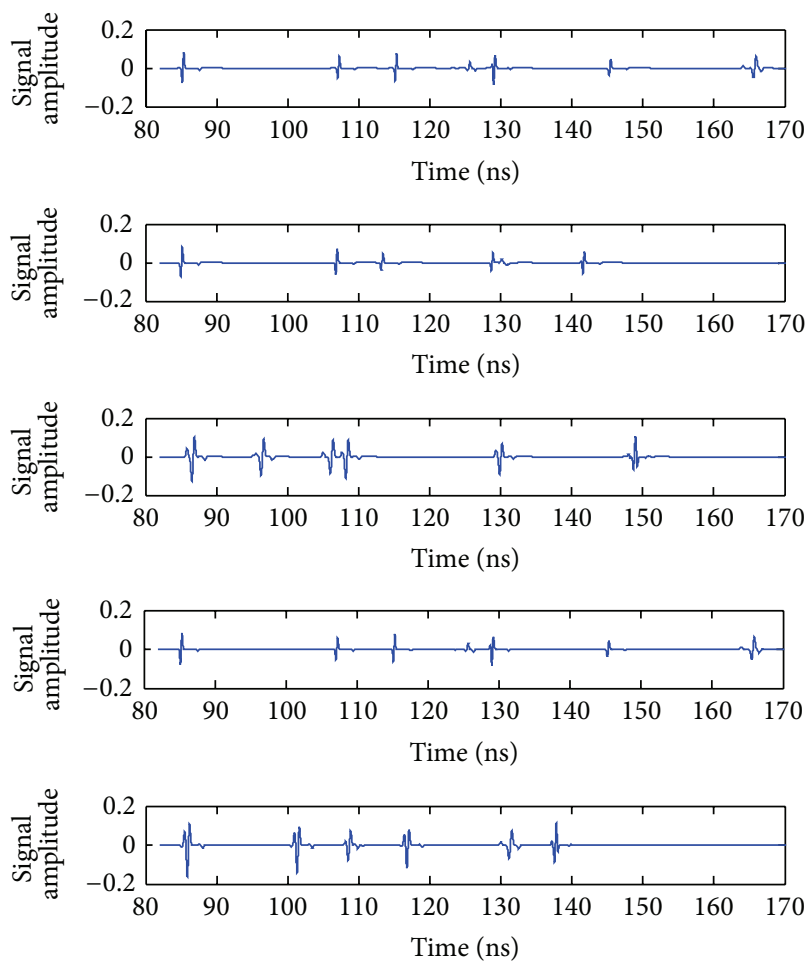

Figure 7: Received signal for the four candidate target positions. True target at $(-6.5,11.5)$.

TABLE 1: Derivation of partial derivatives to evaluate the impact of timing errors on the estimated angle of arrival.

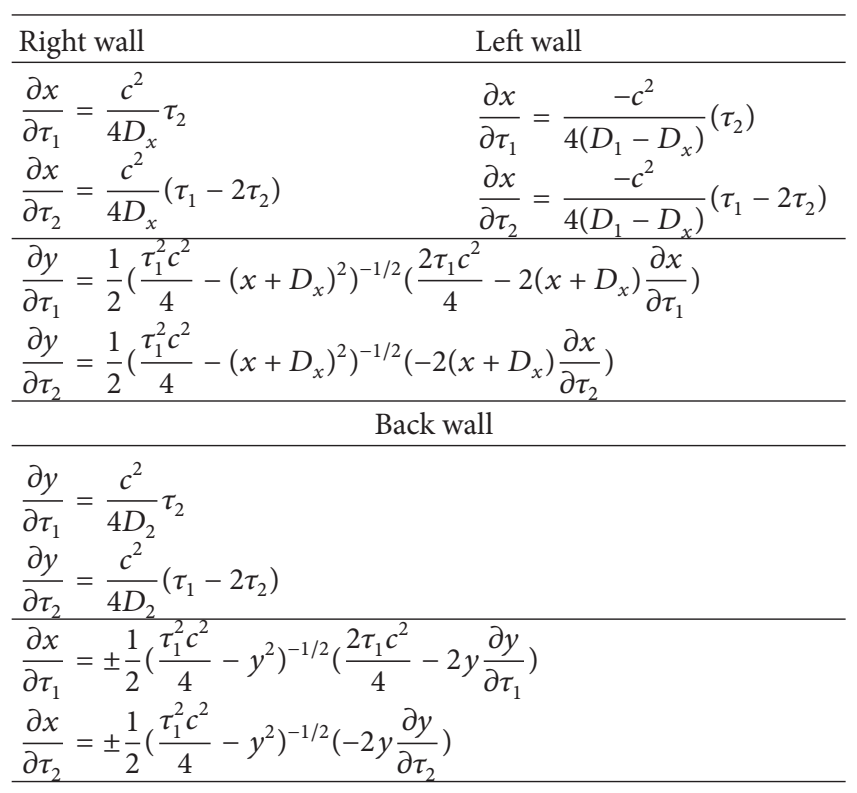

pointing towards $(x+\Delta x, y+\Delta y)$ instead of $(x, y)$. For the example considered in Figure 6 where the radar is located at $(-12,0) \mathrm{m}$ and the target is located at $(-6.5,11.5) \mathrm{m}$, the error in the estimated angle is illustrated in Figure 8. It is shown that an error of $1 \mathrm{~ns}$ in time estimation of both $\tau_{1}$ and $\tau_{2}$ results in less than 5 degrees of error in the angle for the
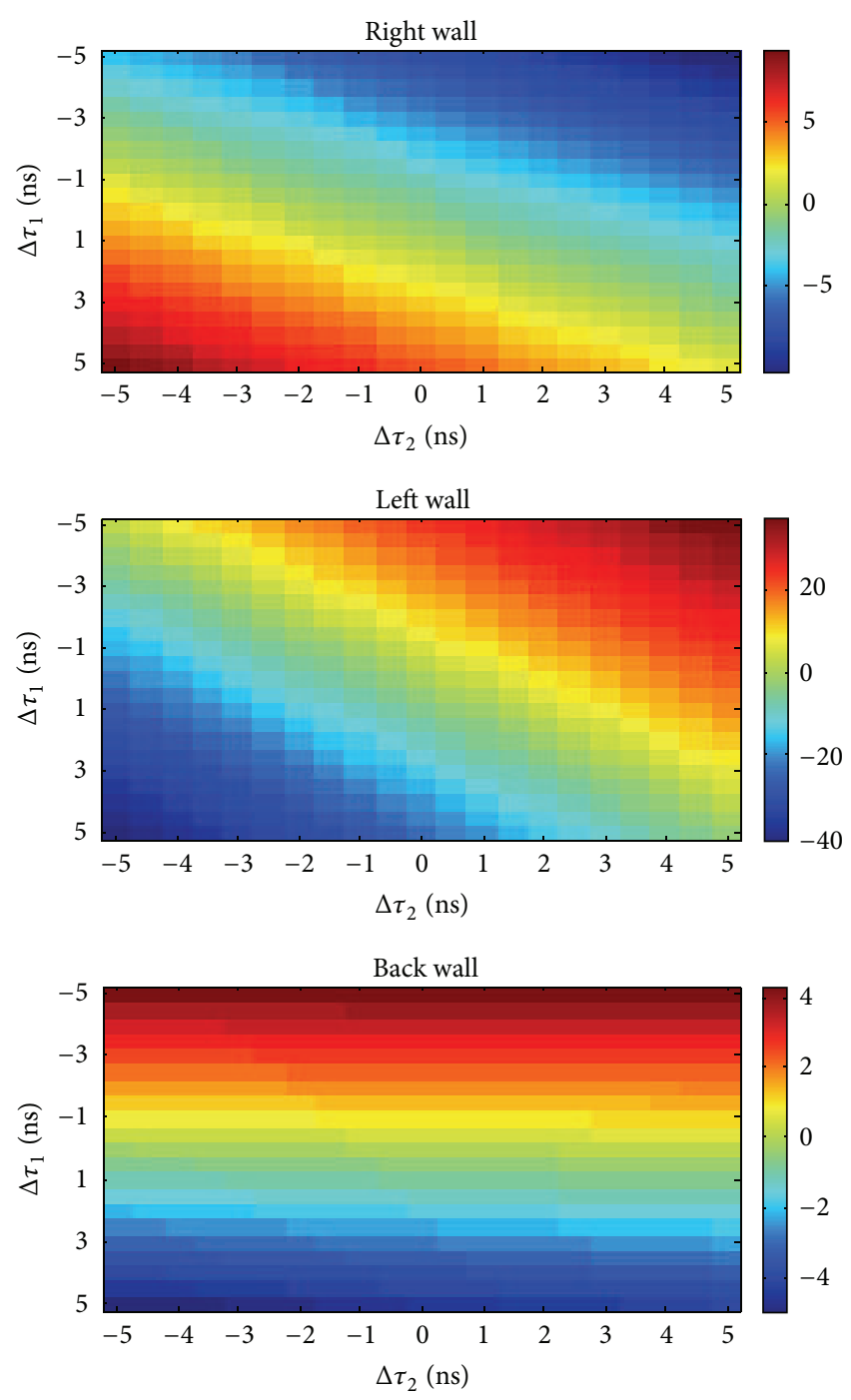

FIGURE 8: Error in angle of arrival (degrees) for the case when the multipath component is due (right, left, back walls).

considered case. It is worth noting that the maximum error occurs when $\Delta \tau_{1}$ has an opposite sign compared with $\Delta \tau_{2}$ when the reflection is from the side walls. When the second arrival is due to the back wall, then the error in the angle is a weak function of $\Delta \tau_{2}$. The third candidate corresponding to a second reflection from the left side wall resulted in the maximum sensitivity to timing error as demonstrated by Figure 8.

\section{Reduction on Localization Error}

In this section we demonstrate the reduction on the error estimation. The exact amount of gain will vary based on the specific localization algorithm.

6.1. Simulation Results. In these simulations, the target is localized using the first two radar returns. Assume the timing error of the multipath components to be independent and Gaussian distributed with variance, $\sigma_{n}^{2}$. For the case of 


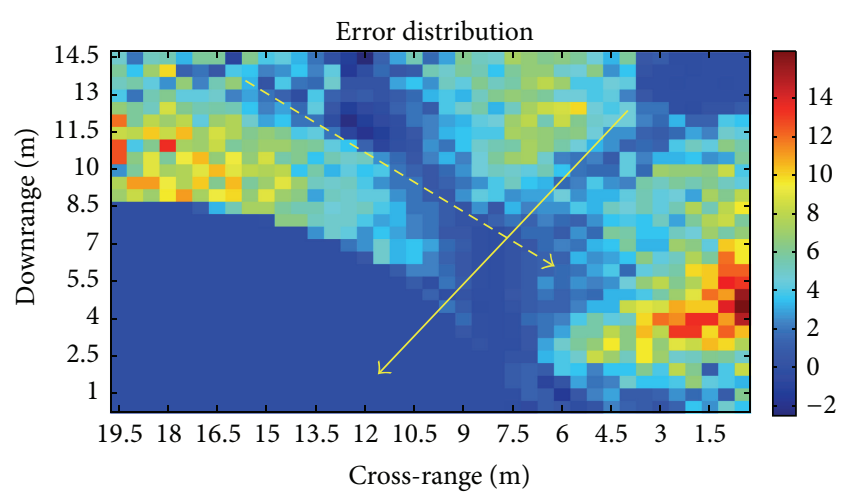

FIgURE 9: Error reduction distribution across the search space.

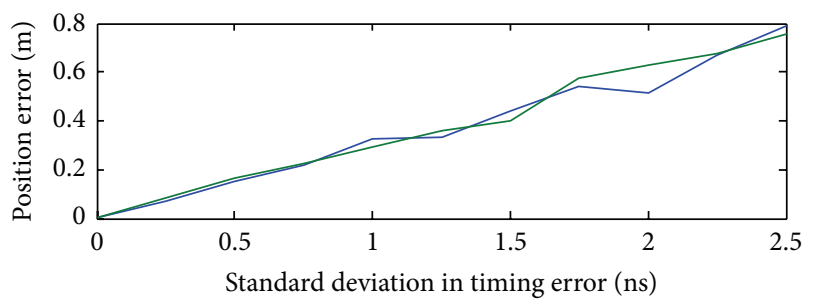

(a)

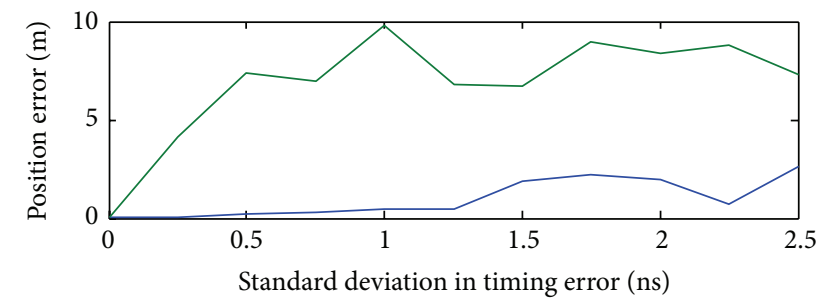

(b)

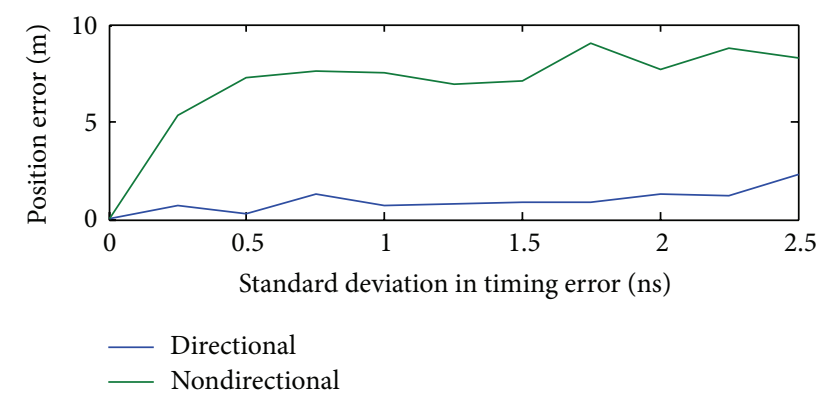

(c)

FIgURE 10: Position error versus timing error at different representative locations ((a) 1 candidate, (b) 3 candidates, and (c) three candidates).

standard deviation, $\sigma_{n}=0.5$, a Monte Carlo simulation was performed. The average localization error defined as the distance between the true target position and the estimated position in meters at various target locations was evaluated for the directional and the nondirectional localization. Figure 9 represents the average reduction in error when directivity is utilized compared with the nondirectional case where the multipath components are all assumed to be of the boresight form. The reduction in localization error is

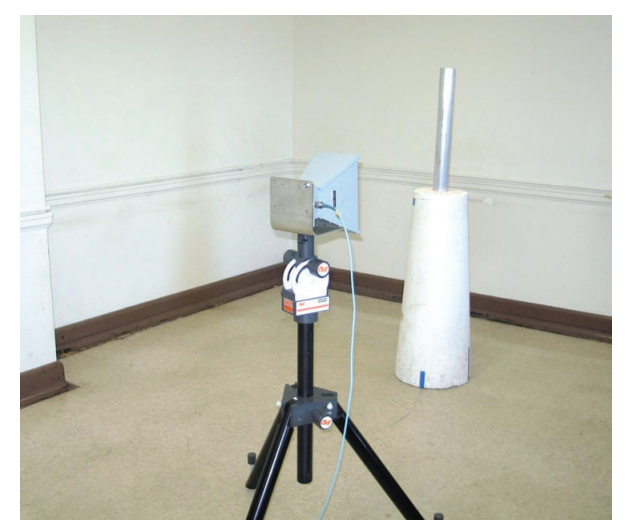

FIGURE 11: Localization experimental environment.

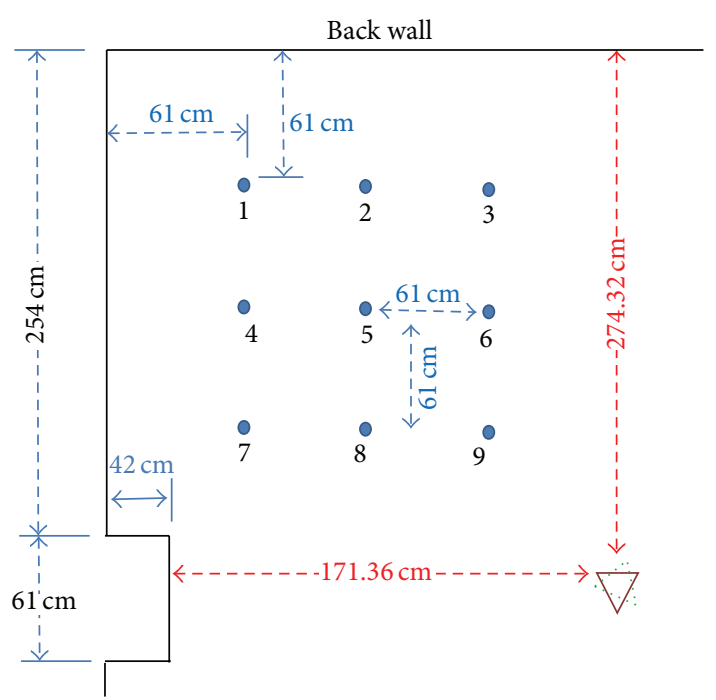

Figure 12: Dimensions and experimental layout.

very evident. Errors may dominate as the target approaches the wall due to solution constraints. These errors can be reduced with conditioning. Other possible sources of large errors include the scenarios where the intersecting circle and ellipse are semitangential. This source of error can be reduced by carefully selecting the location of the sensor.

Comparing Figure 5(a) with Figure 9, we observe that locations with single candidate target result in small localization errors while locations with multiple solutions call for directivity. It is also worth noting that direction of the antenna can be deduced from the plot. The two regions shown by the arrows resulted in small error reduction. The first region (solid arrow) results in a first arrival with boresight shape because antenna is pointing 45 degrees from the $x$-axis. The second arrival (in the region indicated by dashed arrow) has a boresight shape after being reflected from the right side wall.

To examine the performance as a function of the timing error, three possible target locations are selected. The first is $(-14.5,4.5)$ which is a case with only one candidate solution. The positions $(-3.5,4.5)$ and $(-6.5,11.5)$ result in three and four candidates, respectively. Figure 10 provides the variation 

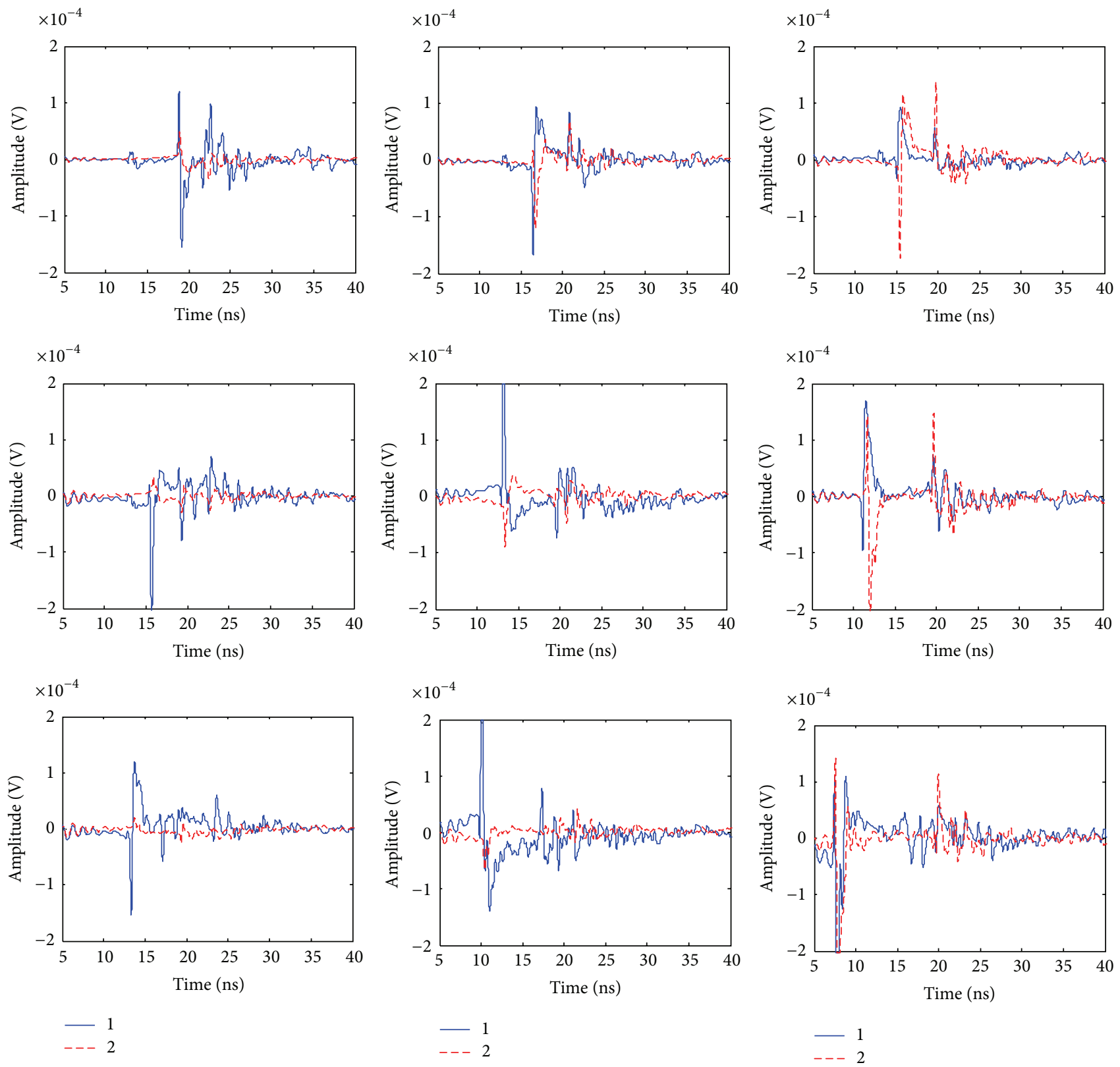

FIGURE 13: Received signal for the two antenna directions at nine different target locations.

of errors as function of the standard deviation of the timing error.

For the case of single candidate, both directional and nondirectional antenna result in a small error of less than 1 meter due to the timing inaccuracy with standard deviation up to 2.5 nanoseconds. However, for the case of multiple possible solutions, directionality resulted in a significant reduction on the positioning error. Note that the errors are location dependent and not a monotonic function.

6.2. Experimental Results. An experimental evaluation was conducted in a classroom at Villanova University, Tolentine Building. Figure 11 depicts the measurement environment. The walls are made of concrete with reinforced concrete columns. A metallic reflector cylinder is used as the target. The diameter of the cylinder is $7.63 \mathrm{~cm}$ while the height is $61 \mathrm{~cm}$. The target is mounted on Styrofoam base with a height of $100 \mathrm{~cm}$. The height of the center of the aperture of the horn antenna is kept at $122 \mathrm{~cm}$. The location of the antenna is shown in Figure 12. The antenna direction was first facing the corner of the room; then, in the second scenario, it was changed to point towards the back wall.

The target position was changed to different locations in the room. Nine selected locations are shown in Figure 12. The target initial location was kept at $61 \mathrm{~cm}$ from the two walls and then moved at steps of $61 \mathrm{~cm}$ in a 3 by 3 grid. An initial measurement was made without the target in place and was subtracted from the subsequent measurements. Hence, all 
the presented waveforms are generated by the target. At every point 32 measurements are averaged to reduce the effect of the ambient noise.

The received signals in the two antenna scenarios are presented in Figure 13. The figure is made of 9 plots representing the 9 different target locations in the same order. Every subplot contains two traces for the two antenna directions to demonstrate the impact of directivity.

Consider the specific case of scenario 1, where the target is located at point 2 . Relative to the reference antenna location, the target is located at $(-91.4,+213.3) \mathrm{cm}$. This target results in multipath arrivals with the direct component round-trip time delay of $15.47 \mathrm{~ns}$ and the second arrival due to the back wall multipath of a corresponding delay of $19.32 \mathrm{~ns}$. These two arrivals could be generated by a target near to location 4, specifically at $(-152.4,152.4) \mathrm{cm}$ relative to the antenna, with the second reflection generated by the side wall. To decide between the two locations, correlation is performed between the received profile and the expected respective profile. The expected profiles are generated using a directional ray-tracing simulator. The ray-tracing simulator determines the expected multipath components and their magnitudes and angles and builds the candidate profiles based on the known scene geometry. Due to the limited number of candidates and the expected differences in their profiles in terms of times and directions of arrival, even in the presence of some noise and timing jitter, the receiver will be able to select best the candidate. The results support the selection of position number 2. Due to the horizontal polarization of the antenna, the second arrival is inverted when comparing location 2 with location 4. Refer to trace 1 in the second and fourth plot in Figure 12 at the time of the second arrival $19.32 \mathrm{~ns}$. The difference is due to the fact that the second radar return in case 2 is due to the back wall, whereas in case 2 it is due to the side wall. The two multipath returns result in different angles of arrival. A similar polarity observation can be made when comparing the direct arrival in case 4 and case 5 .

\section{Conclusion}

In this paper, we introduced a new approach for indoor target localization exploiting multipaths from surrounding walls. This approach uses pulse shape dependency on DOA of the radar return for excluding false target locations. Solutions of target position coordinates can proceed using only two multipaths. This capability becomes important when exploitation of all possible multipaths fails due to obstruction or weak signal detection. Further, applying the proper angledependent matched filter at the receiver in lieu of the nominal filter corresponding to boresight increases signal-to-noise ratio and as such reduces timing errors. The effectiveness of our approach was supported by both simulations and measurements.

\section{Conflict of Interests}

The authors declare that there is no conflict of interests regarding the publication of this paper.

\section{Acknowledgment}

The work by Dr. Ali H. Muqaibel was supported by the Deanship of Scientific Research at King Fahd University of Petroleum and Minerals (KFUPM).

\section{References}

[1] B. D. Rigling, "Urban RF multipath mitigation," IET Radar, Sonar and Navigation, vol. 2, no. 6, pp. 419-425, 2008.

[2] J. L. Krolik, J. Farrell, and A. Steinhardt, "Exploiting multipath propagation for GMTI in urban environments," in Proceedings of the IEEE Radar Conference, pp. 65-68, April 2006.

[3] Y. Kuang, K. Astrom, and F. Tufvesson, "Single antenna anchorfree UWB positioning based on multipath propagation," in Proceedings of the IEEE International Conference on Communications (ICC '13), pp. 5814-5818, IEEE, Budapest, Hungary, June 2013.

[4] D. Deiana, A. S. Kossen, and W. L. van Rossum, "Multipath exploitation in an urban environment using a MIMO surveillance radar," in Proceedings of the 11th International Radar Symposium (IRS '10), pp. 178-181, June 2010.

[5] S. Sen and A. Nehorai, "Adaptive OFDM radar for target detection in multipath scenarios," IEEE Transactions on Signal Processing, vol. 59, no. 1, pp. 78-90, 2011.

[6] B. Chakraborty, Y. Li, J. J. Zhang, T. Trueblood, A. PapandreouSuppappola, and D. Morrell, "Multipath exploitation with adaptive waveform design for tracking in urban terrain," in Proceedings of the IEEE International Conference on Acoustics, Speech, and Signal Processing (ICASSP '10), pp. 3894-3897, March 2010.

[7] L. B. Fertig, M. J. Baden, J. C. Kerce, and D. Sobota, "Localization and tracking with multipath exploitation radar," in Proceedings of the IEEE Radar Conference: Ubiquitous Radar (RADARCON '12), pp. 1014-1018, Atlanta, Georgia, USA, May 2012.

[8] P. Setlur, M. Amin, and F. Ahmad, "Multipath model and exploitation in through-the-wall and urban radar sensing," IEEE Transactions on Geoscience and Remote Sensing, vol. 49, no. 10, pp. 4021-4034, 2011.

[9] M. Leigsnering, F. Ahmad, M. Amin, and A. Zoubir, "Multipath exploitation in through-the-wall radar imaging using sparse reconstruction," IEEE Transactions on Aerospace and Electronic Systems, vol. 50, no. 2, pp. 920-939, 2014.

[10] S. Kidera, T. Sakamoto, and T. Sato, "Experimental study of shadow region imaging algorithm with multiple scattered waves for UWB radars," PIERS Online, vol. 5, no. 4, pp. 393-396, 2009.

[11] S. Kidera, T. Sakamoto, and T. Sato, "Extended imaging algorithm based on aperture synthesis with double-scattered waves for UWB radars," IEEE Transactions on Geoscience and Remote Sensing, vol. 49, no. 12, pp. 5128-5139, 2011.

[12] G. Gennarelli and F. Soldovieri, "A linear inverse scattering algorithm for radar imaging in multipath environments," IEEE Geoscience and Remote Sensing Letters, vol. 10, no. 5, pp. 10851089, 2013.

[13] G. Smith and B. Mobasseri, "Multipath exploitation for radar target classification," in Proceedings of the Radar Conference (RADAR '12), IEEE, 2012.

[14] P. Setlur, G. E. Smith, F. Ahmad, and M. G. Amin, "Target localization with a single sensor via multipath exploitation," IEEE Transactions on Aerospace and Electronic Systems, vol. 48, no. 3, pp. 1996-2014, 2012. 
[15] A. H. Muqaibel and U. M. Johar, "UWB multipath simulator based on TEM horn antenna," in Proceedings of the 2nd International Conference on Wireless Broadband and Ultra Wideband Communications (AusWireless '07), p. 9, Sydney, Australia, August 2007.

[16] A. Attiya and A. Safaai-Jazi, "Time domain characterization of receiving TEM horn antennas," in Proceedings of the IEEE Antennas and Propagation Society International Symposium, vol. 1, pp. 233-236, Columbus, Ohio, USA, 2003.

[17] G. S. Smith, "Teaching antenna reception and scattering from a time-domain perspective," American Journal of Physics, vol. 70, no. 8, pp. 829-844, 2002. 

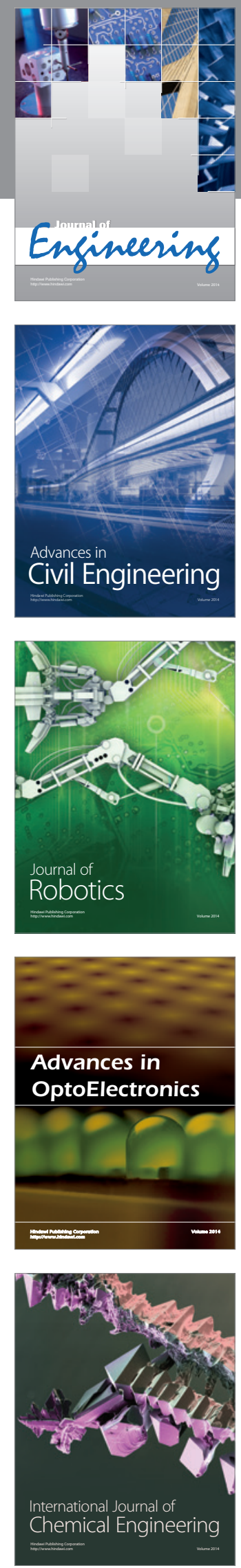

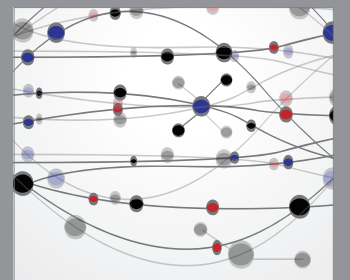

The Scientific World Journal
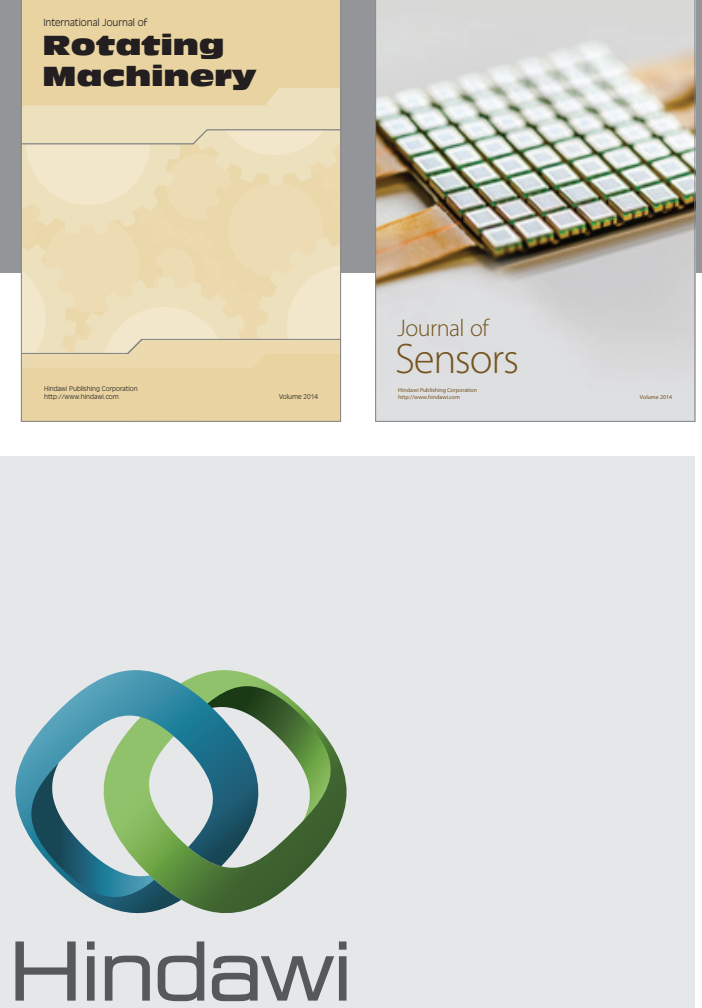

Submit your manuscripts at http://www.hindawi.com
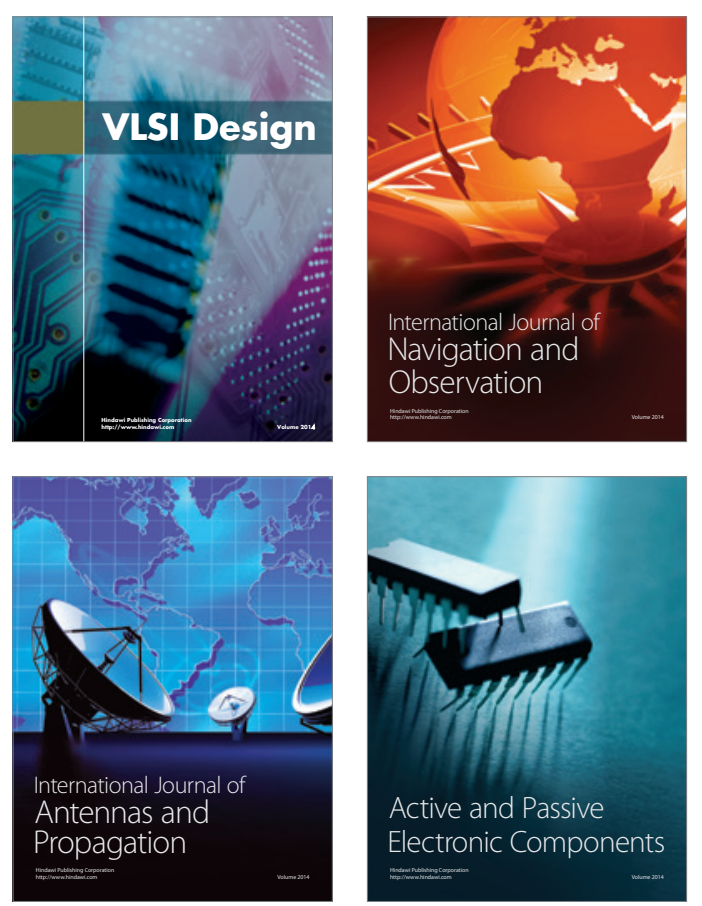
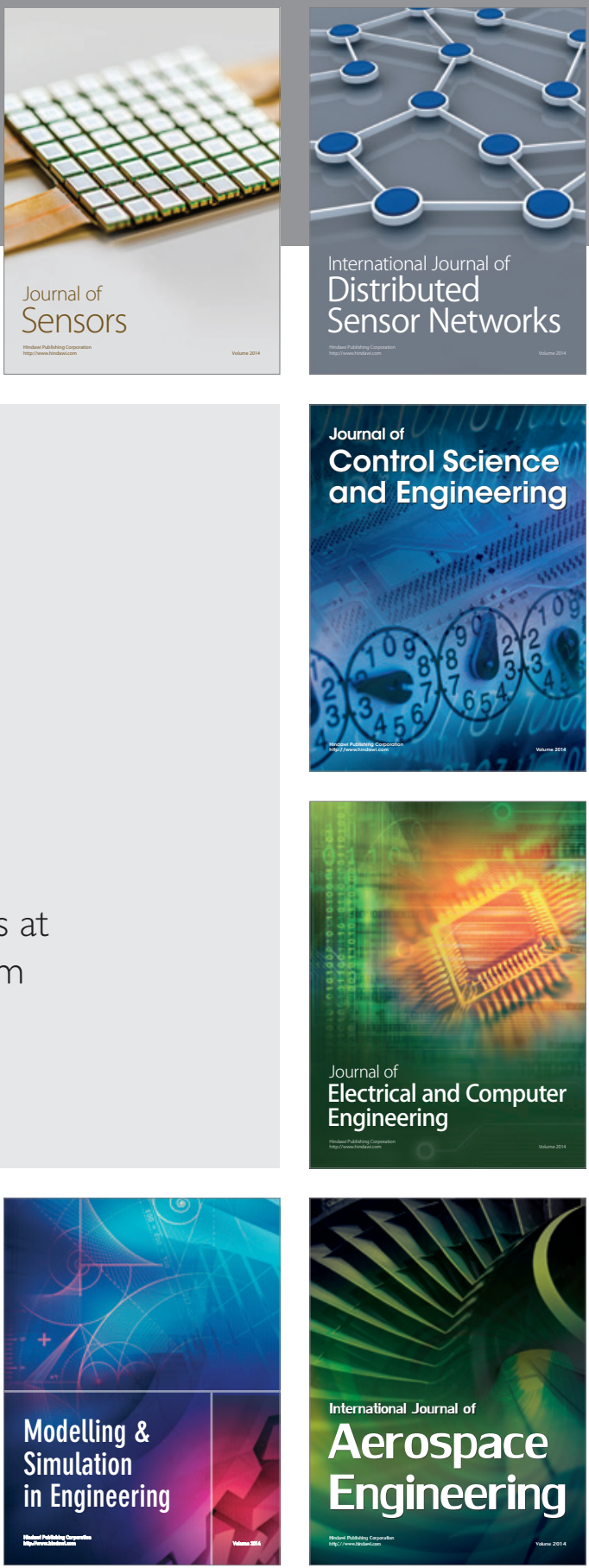

Journal of

Control Science

and Engineering
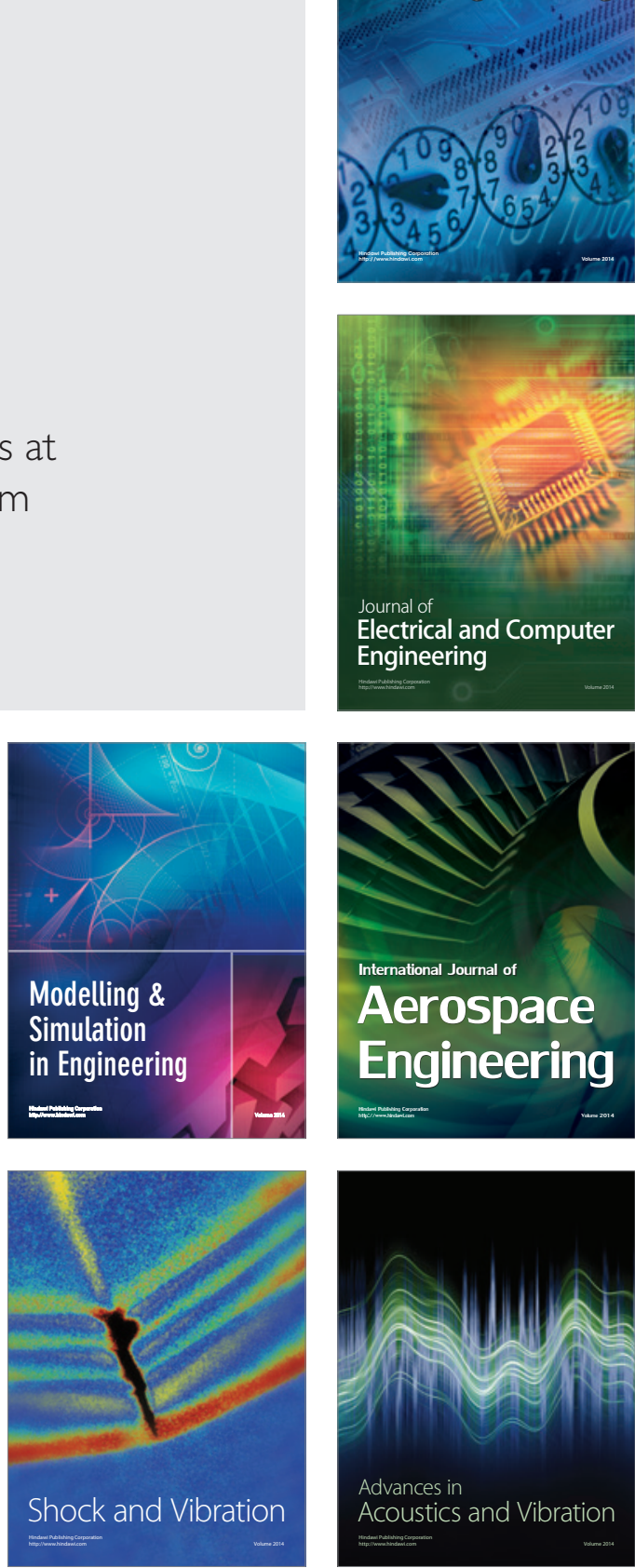